\title{
Losses of Chromosomal Arms 1p and 19q in the Diagnosis of Oligodendroglioma. A Study of Paraffin- Embedded Sections
}

Peter C. Burger, M.D., A. Yuriko Minn, M.S., Justin S. Smith, M.D., Ph.D., Thomas J. Borell, B.S., Anne E. Jedlicka, M.S., Brenda K. Huntley, B.S., Patricia T. Goldthwaite, M.S.,

Robert B. Jenkins, M.D., Ph. D., Burt G. Feuerstein, M.D., Ph.D.

The Departments of Pathology (PCB, PTG) and Anesthesiology and Critical Care Medicine (AEJ), Johns Hopkins University School of Medicine, Baltimore, Maryland; Department of Pathology and Laboratory Medicine, Mayo Clinic (JSS, TJB, BKH, RBJ), Rochester, Minnesota; and Departments of Laboratory Medicine and Neurosurgery and Brain Tumor Research Center, University of California at San Francisco School of Medicine (BGF, AYM), San Francisco, California

Comparative genomic hybridization (CGH), fluorescence in situ hybridization (FISH), polymerase chain reaction-based microsatellite analysis, and p53 sequencing were performed in paraffin-embedded material from 18 oligodendrogliomas and histologically similar astrocytomas. The study was undertaken because of evidence that concurrent loss of both the 1p and 19q chromosome arms is a specific marker for oligodendrogliomas. Of the six lesions with a review diagnosis of oligodendroglioma, all had the predicted loss of $1 \mathrm{p}$ and $19 \mathrm{q}$ seen by CGH, FISH, and polymerase chain reaction. Other lesions, including some considered oligodendroglioma or mixed glioma by the submitting institution, did not. There were no p53 mutations in any of the six oligodendrogliomas, whereas 5 of the 10 remaining, successfully studied cases did have p53 mutations. The results suggest that CGH and FISH performed on current or archival tissue can aid in classification of infiltrating gliomas such as oligodendrogliomas and astrocytomas. The results of the p53 studies are consistent with findings of previous investigations that such mutations are less common in oligodendrogliomas than they are in astrocytomas.

KEY WORDS: Astrocytoma, Comparative genomic hybridization, FISH, Genetics, Oligodendroglioma.

Mod Pathol 2001;14(9):842-853

Copyright (C) 2001 by The United States and Canadian Academy of

Pathology, Inc.

VOL. 14, NO. 9, P. 842, 2001 Printed in the U.S.A.

Date of acceptance: March 19, 2001.

Supported by the following NIH grants to the Glioma Marker Network: CA64928, CA50905, and CA64898.

Address reprint requests to: Peter C. Burger, M.D., Department of Pathology, The Johns Hopkins Hospital, 600 N. Wolfe Street, Baltimore, MD 21287; e-mail: pburger@jhmi.edu; fax: 410-614-9310.
It is the impression of some pathologists that many gliomas presently designated as oligodendrogliomas would have been classified in the past as astrocytomas with little thought about the possibility of an oligodendroglial component. It also appears that the relative incidence of the diagnoses of oligodendroglioma and astrocytomas varies widely from institution to institution, suggesting that diagnostic criteria differ. In some laboratories, even minor or focal nuclear roundness or perinuclear haloes are considered to be evidence of oligodendroglial differentiation, whereas pathologists elsewhere require the more classical features. Perhaps even more important, many infiltrating gliomas are difficult to categorize on the basis of pathological features. There is clearly a need for objective criteria to resolve a question increasingly asked of surgical pathologists- - Is this neoplasm oligodendroglioma or mixed glioma?" In large part, this need to classify a tumor as "oligodendroglial" is motivated by the general perception that oligodendrogliomas are less intrinsically aggressive and more responsive to therapy than are infiltrating astrocytomas of comparable grade (1).

There is abundant evidence that combined loss of the short arm of chromosome 1 (1p) and the long arm of chromosome 19 (19q) is a signature of oligodendrogliomas (2-12). Although it is not clear whether all oligodendrogliomas are so marked or what the nosology should be for oligodendrogliomas without the $1 \mathrm{p} / 19 \mathrm{q}$ loss, the association between classic oligodendroglial histopathological features and a combined $1 \mathrm{p}$ and $19 \mathrm{q}$ loss is likely to have biological significance and diagnostic utility. An important question for the surgical pathologist is whether $1 \mathrm{p} / 19 \mathrm{q}$ loss is a useful diagnostic test for individual cases. The present study addressed these issues by correlating histological and immunohis- 
tochemical findings in a series of oligodendrogliomas, astrocytomas, and "mixed gliomas" with genetic information from three techniques applied to paraffin-embedded tissue, that is, comparative genomic hybridization (CGH), fluorescence in situ hybridization (FISH), and polymerase chain reaction (PCR)-based microsatellite analysis for loss of heterozygosity (LOH).

A second component of the study addressed the issue of p53 mutations. Such mutations, as defined by DNA sequencing, are infrequent in welldifferentiated oligodendrogliomas $(6,13-15)$, although immunohistochemical reactivity for p53 is not uncommon $(16,17)$. In "mixed" oligoastrocytomas, p53 mutations have been found in a subset without the signature $1 \mathrm{p} / 19 \mathrm{q}$ loss. All of these observations raise the question of whether p53 mutations are present in oligodendrogliomas rigidly defined by both histologic and cytogenetic/molecular criteria.

\section{MATERIALS AND METHODS}

\section{Case Selection}

Hematoxylin and eosin (H\&E)-stained sections and paraffin blocks were collected from 18 cases of classic and borderline examples of infiltrating gliomas of oligodendroglioma, mixed gliomas (oligoastrocytomas), fibrillary astrocytomas of varying grade, and difficult-to-classify intermediate lesions (Table 1). Fourteen of the lesions had been diagnosed as either oligodendroglioma or "mixed" oligoastrocytoma. Two malignant gliomas had abundant "minigemistocytes," raising the issue of an oligodendroglial component. The sections were reviewed by one of us (PCB) and the neoplasms classified as oligodendroglioma Grade II or Grade III, fibrillary astrocytoma Grade II, III, or IV (glioblas- toma multiforme), or malignant mixed glioma Grade III. This review was done without any knowledge of the CGH, FISH, or PCR findings. In one case, T107, DNA isolated from fresh tumor was available and was used for the cytogenetic, FISH, and LOH studies rather than was the DNA from the paraffin-embedded tissue.

The histological criteria for the review diagnoses, along the general guidelines of the World Health Organization (18), were as follows:

1. Oligodendroglioma Grade II A lesion composed of strikingly uniform cells with distinctly round nuclei and prominent perinuclear halos giving the classic fried-egg appearance. Although the lesion infiltrates the parenchyma, solid areas with little if any detectable intercellular parenchyma were often present.

2. Oligodendroglioma Grade III A tumor with similar features to the Grade II lesion but with higher cellularity and more cytological atypia. Scattered mitoses and foci of vascular proliferation were required.

3. Fibrillary astrocytoma Grade II An infiltrating lesion with more hyperchromatic and irregular nuclei than those of oligodendroglioma Grade II. Perinuclear haloes, sometimes prominent, were permitted, but none of the lesions had the monomorphism and extensive fried-egg appearance of an oligodendroglioma. A solitary mitosis was permitted. Solid areas of back-to-back cells were not present.

4. Fibrillary astrocytoma Grade III A glioma similar to the Grade II astrocytoma but with higher cellularity and cytological atypia. Two or more mitoses are required. Neither vascular proliferation nor necrosis was permitted. Perinuclear haloes were accepted, but the lesions could not have the

\section{TABLE 1.}

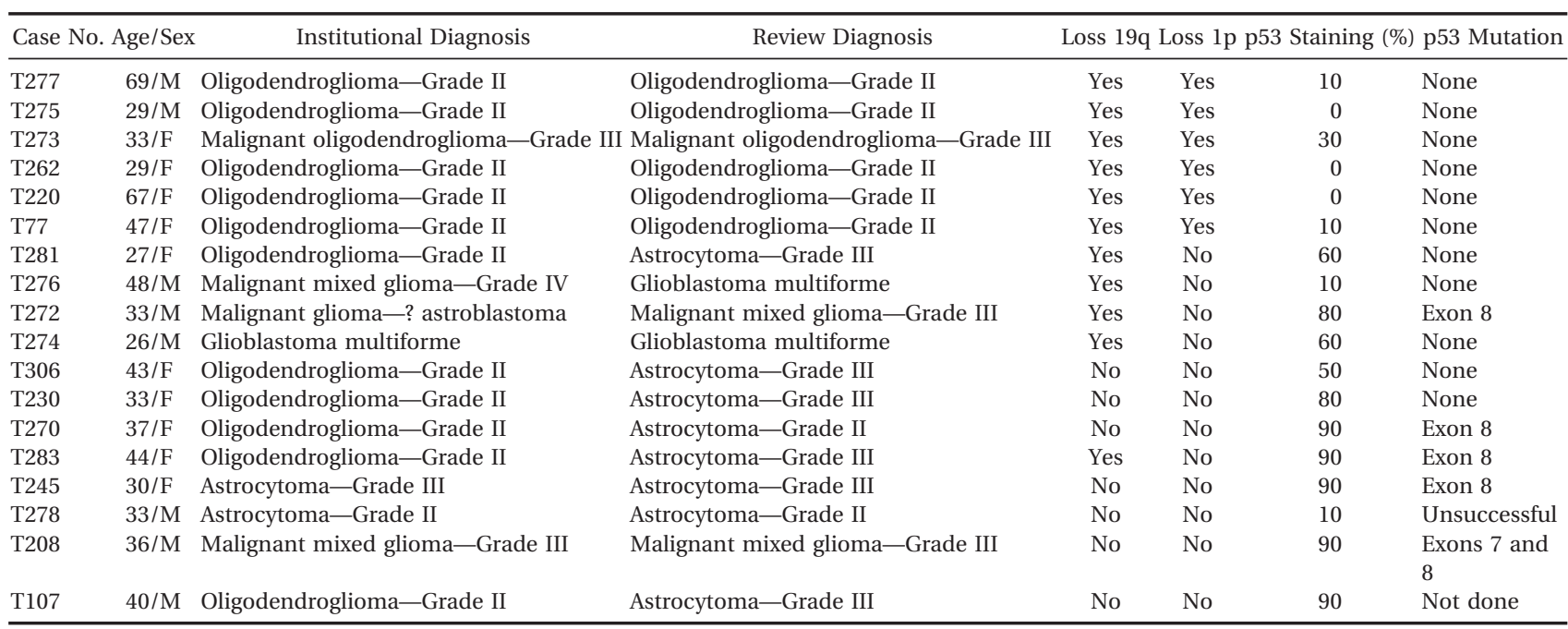


cellular monomorphism and uniform high cellularity of the Grade III oligodendroglioma.

5. Mixed malignant glioma Grade III A lesion with areas that resembled both oligodendroglioma and fibrillary astrocytoma Grade III.

6. Glioblastoma multiforme The classical lesion with pleomorphic cells in association with necrosis and/or vascular proliferation.

For each case, $10-\mu \mathrm{m}$ sections were cut and mounted on unstained slides. Microdissection was not done, although in Case T276, two blocks were cut and studied separately. An H\&E-stained section was prepared and examined to be certain that tissue contained $\geq 60 \%$ tumor cells. An adjacent section was stained immunohistochemically for p53 protein as described later in this article.

\section{Immunohistochemistry for p53}

Immunohistochemical staining for p53 was performed using DO-7 antibody (Vector Inc., Burlingame, CA). The antibody recognizes both normal and mutated forms of the protein. The deparaffinized slides were subjected to antigen retrieval by microwaving in $10 \mathrm{~mm}$ citrate buffer. The antibody was recognized with the avidin-biotin complex method, with diaminobenzidine as the chromogen.

Given the regional variability in staining, a semiquantitative measure of staining frequency was used that designated the approximate percentage of tumor cell nuclei that were positive, in deciles.

\section{Comparative Genomic Hybridization}

Tumor DNA was extracted from three to five $10-\mu \mathrm{m}$ sections of paraffin-embedded tissue, depending on the size of the sections. All were $\geq 1 \mathrm{~cm}$ in shortest dimension. Tissue was deparaffinized in xylene at $55^{\circ} \mathrm{C}$, washed in ethanol, and then washed in phosphate buffered saline. Tumor cells were resuspended in a nuclear lysing buffer $(25 \mathrm{~mm}$ EDTA, $100 \mathrm{~mm} \mathrm{NaCl}$, and $10 \mathrm{~mm}$ Tris) and treated with proteinase $\mathrm{K}(500 \mathrm{ug} / \mathrm{mL})$ and $0.5 \%$ SDS for 3 days at $55^{\circ} \mathrm{C}$. DNA was extracted with phenolchloroform-isoamyl alcohol (25:24:1), precipitated with $10 \mathrm{~m}$ ammonium acetate, $100 \%$ ethanol, and resuspended in TE buffer (19). Reference DNA was prepared from leukocytes of normal male and female donors.

Target DNA was amplified by adapting a protocol for degenerate oligonucleotide-primed PCR (DOPPCR) described by Telenius et al. (20). The first five cycles of annealing and extension were done at a low temperature using Sequenase (Amersham, Arlington Heights, IL) to maximize genome representation. This was followed by a high stringency reaction using Taq polymerase (Boehringer Mannheim, Indianapolis, IN). DNA was labeled with either fluorescein isothiocyanate (FITC)-12deoxyuridine triphosphate (dUTP; DuPont, Wilmington, DE) or digoxigenin-11 dUTP (Boehringer Mannheim) by taking $5 \mu \mathrm{L}$ of the original DOP-PCR product and performing 25 PCR cycles with degenerate primers and Taq polymerase. Probe fragment size (optimal range, 300 to 2000 base pairs) was determined by agarose electrophoresis.

CGH analysis was performed as described by Mohapatra et al. in 1995 (21). Normal metaphase spreads were prepared from phytohemagglutininstimulated peripheral blood lymphocytes obtained from a healthy normal male donor by using standard procedures or were obtained from Vysis Inc. (Downers Grove, IL). Digoxigenin-labeled tumor DNA (200 ng), and FITC-labeled normal DNA (200 ng) and Cot-1 DNA (20 $\mu \mathrm{g}$; Life Technologies, Gaithersburg, MD) were ethanol precipitated and resuspended in $10 \mu \mathrm{L}$ of $50 \%$ formamide, $10 \%$ dextran sulfate, and $2 \times$ SSC. The probe mixture was denatured at $80^{\circ} \mathrm{C}$ and hybridized to normal human metaphase spreads, then incubated at $37^{\circ} \mathrm{C}$ for 48 hours. Slides were washed at $45^{\circ} \mathrm{C}$ in $50 \%$ formamide $/ 2 \times$ SSC and $2 \times$ SSC and at room temperature in $2 \times$ SSC. Digoxigenin-labeled DNA was stained with anti-digoxigenin rhodamine (1 $\mu \mathrm{g} / \mathrm{mL}$ ). The slides were washed again in $4 \times$ SSC, $4 \times$ SSC $/ 0.1 \%$ Triton, $4 \times$ SSC, and water. Slides were counterstained with 0.2 uM DAP1 in an antifade solution.

CGH image analysis was performed as described by Piper et al. (22). Slides were viewed with a Zeiss Axioplan fluorescence microscope (Zeiss, Thornwood, NY). Red, green, and blue images were collected using a Quantitative Image Processing System. Analyses of green to red ratios for each chromosome were done using Quips CGH Analysis Software (Vysis Inc.). At least eight copies of each autosome and four copies of each sex chromosome were visually inspected and analyzed for each tumor set. Only those chromosomes with uniform hybridization were used for analysis. Amplifications in the genome were visualized as discrete subchromosomal bands. Copy number aberrations were scored if the relative gain was $>1.2$ and if the relative loss was $<0.8(21)$.

\section{PCR-Based Microsatellite Analysis}

\section{Isolation of DNA}

DNA for microsatellite analysis was isolated using five paraffin sections prepared for FISH and a microwave-based extraction method (23). Briefly, tumor tissue from corresponding regions enumerated by FISH was scraped from the glass slides and transferred into $1.5-\mathrm{mL}$ sterile microcentrifuge tubes. The tissue was gently crushed in $200 \mathrm{~mL}$ of digestion buffer (50 mM Tris- $\mathrm{HCl}, \mathrm{pH} 8.5 ; 1 \mathrm{~mm}$ 
EDTA; and $0.5 \%$ Tween $20^{\circledR}$; Sigma Chemical, St. Louis, MO). The tubes were capped and subjected to microwave irradiation at high power $(500 \mathrm{~W})$ for 30 to 60 seconds. After microwave treatment, the samples were centrifuged $(12,000 \mathrm{rpm}$ for $10 \mathrm{~min}$ at room temperature), producing a readily extractable paraffin ring above the buffer The remaining tissue pellet was resuspended in digestion buffer, $2.4 \mu \mathrm{L}$ of proteinase $\mathrm{K}$ was added to each tube, and samples were digested for 120 hours at $42^{\circ} \mathrm{C}$. After digestion, the tubes were centrifuged for 5 minutes at $6000 \mathrm{rpm}$. The DNA-containing supernatant was pipetted into a new sterile tube and boiled for 10 minutes to denature any residual protease and contaminating proteins.

\section{Microsatellite Analysis}

Microsatellite markers spanning the length of both the $1 p$ and $19 q$ chromosome arms were selected based on amplicon size and heterozygosity score. Selected microsatellite markers were placed in their most probable linkage order based on radiation hybrid and physical mapping (24-27) and were amplified from 18 tumor specimens and 8 unrelated control lymphocyte DNA specimens. PCR assays were performed with the following: approximately $1 \mathrm{ng}$ DNA, $50 \mathrm{~mm}$ Tris-C1 (pH 8.0), $100 \mathrm{~mm}$ $\mathrm{KC} 1,1.5$ to $3.0 \mathrm{~mm} \mathrm{MgCl}_{2}, 0.1$ to $0.3 \mu \mathrm{L}$ of $(\alpha-$ $\left.{ }^{32} \mathrm{P}\right) \mathrm{dCTP}(3000 \mathrm{Ci} / \mathrm{mm}$, NEN, Boston, MA), $33 \mu \mathrm{M}$ dCTP, $100 \mu \mathrm{M}$ dNTP (dATP, dGTP, dTTP), 2 pм of each primer, and $0.5 \mathrm{U}$ of Amplitaq Gold (PerkinElmer, Foster City, CA). DNA was PCR amplified in a Perkin-Elmer 9600 thermal cycler. After PCR amplification, samples were denatured at $95^{\circ} \mathrm{C}$ in an equal volume of denaturing buffer containing formamide quenched on ice, and 2 to $4 \mu \mathrm{L}$ were applied to thick, $6 \%$ denaturing sequencing gels. Gels were electrophoresed at $75 \mathrm{~W}$ at room temperature, captured on Whatman paper, dried, and exposed for 4-24 hours to single-sided Kodak emulsion film. Autoradiographs were visually inspected, and amplicons were scored as heterozygous, homozygous, or indeterminate.

\section{Fluorescence In Situ Hybridization}

Five bacterial artificial chromosomes (BACs) were labeled to generate locus-specific FISH probes (LSFP) mapping to 1p36, 1q24, 19p13.1-q13.1q13.2, and 19q13.3. The BAC mapping to $19 \mathrm{q} 13.3$ was selected on the basis of previous reports that this band contains a region of common allelic loss in gliomas $(2-5,7,9-11,28,29)$. The BAC mapping to $19 q 13.1-q 13.2$ was selected using primers for the AKT2 gene.

FISH analysis was performed on paraffinembedded tissue as previously described $(30,31)$. Briefly, approximately ten to fifteen $5-\mu \mathrm{m}$ sections were prepared from paraffin-embedded blocks for each tumor. The first and last sections were $H \& E$ stained and the regions representing tumor and normal tissues delineated. Tissue sections were deparaffinized, dehydrated, treated with microwave in citrate buffer ( $\mathrm{pH}$ 6.0) for 10 minutes, digested in pepsin solution ( $4 \mathrm{mg} / \mathrm{mL}$ in $0.9 \% \mathrm{NaCl}, \mathrm{pH} 1.5$ ) for 15 minutes at $37^{\circ} \mathrm{C}$, rinsed in $2 \times \mathrm{SSC}$ at room temperature for 5 minutes, and air dried. Dualprobe hybridization was performed using a digoxigenin (DIG)-labeled locus-specific $1 p$ or $19 q$ probe that was detected with rhodamine and a Spectrum Green-labeled probe mapping to $1 \mathrm{q}$ and 19p. Probes and target DNA were denatured simultaneously in an $80^{\circ} \mathrm{C}$ oven for 5 minutes, followed by overnight incubation at $37^{\circ} \mathrm{C}$. Slides were then washed in $1.5 \mathrm{M}$ urea $/ 0.1 \times \mathrm{SSC}$ at $45^{\circ} \mathrm{C}$ for 30 minutes and in $2 \times$ SSC at room temperature for 2 minutes. After washing, the DIG-labeled probes were detected using a rhodamine detection kit (Oncor, Gaithersburg, MD). Nuclei were counterstained with 4,6-diamidino-2 phenylindole and the antifade compound p-phenylenediamine.

A Zeiss Axioplan microscope equipped with a triple-pass filter (DAPI/Green/Orange; Vysis, Downers Grove, IL) was used to assess the number of FIS signals for each LSFP. Approximately 300 nonoverlapping nuclei were enumerated per hybridization.

\section{Sequencing of p53}

Exons 5 through 8 were amplified from DNA isolated from unstained paraffin-embedded sections that had been mounted on glass slides. The sequencing techniques were those described by Brat et al. (32).

\section{RESULTS}

Histological Findings and Tumor Classification and Grading

\section{Oligodendrogliomas}

T262 A classic very well differentiated oligodendroglioma with back-to- back cells with perinuclear haloes (Fig. 1A). There were also areas of infiltration where the cells were more widely spaced (Fig. 1B). Haloes were not as prominent in these areas.

T220 A classic, very well-differentiated oligodendroglioma with back-to-back cells with haloes (Fig. 1C), plus focal areas with "minigemistocytes" with a small amount of eosinophilic cytoplasm (Fig. 1D).

T277 Focally a paucicellular Grade II example, but overall, with the cellularity that approached 

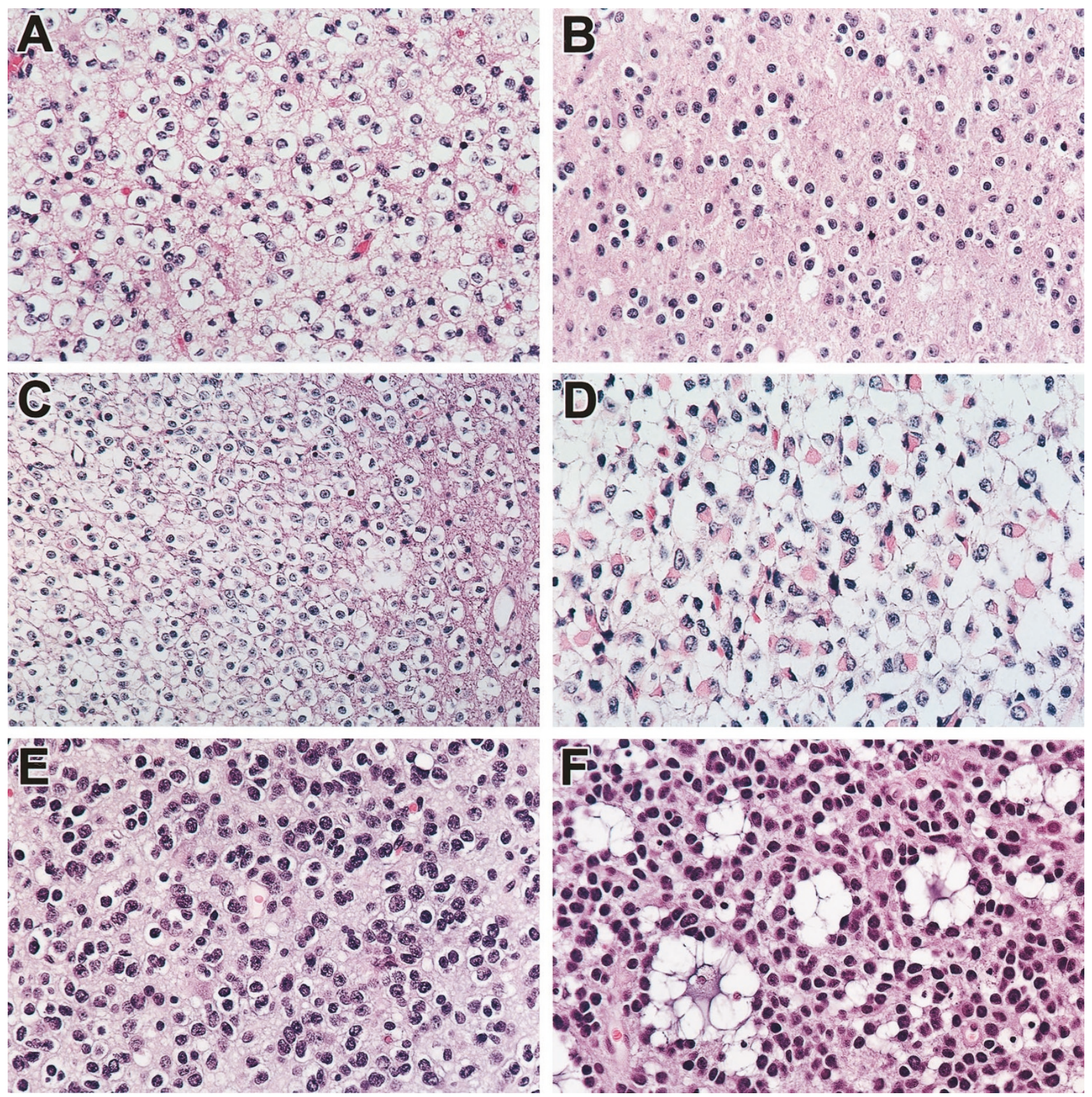

FIGURE 1. Histological features of representative cases with combined loss of chromosomal arms 1p and 19q. A and B, Case T262. (A) illustrates classic histopathological features of oligodendroglioma, in other words, cellular monotony and perinuclear haloes. The diagnosis was not as elementary elsewhere in the same lesion because cellularity was lower and haloes were less pronounced (B). C and D, Case T220. The tumor in most areas had classic features of oligodendroglioma (C) but elsewhere was composed of cells with prominent eosinophilic cytoplasm (D). These cells, which were GFAP positive, retained the round nuclei expected of oligodendrocytes. E and F, Case T277. The cellular lesion retained a general nuclear roundness and perinuclear haloes expected of an oligodendroglioma (E). Microcystic change was prominent (F).

that of Grade III but lacked vascular proliferation (Fig. 1E-F). Microcysts, calcification, subpial accumulation, and nodules of higher cellularity were present. The nuclei in the more cellular areas were dense but less atypical than those in T273.

T275 A classic, very well differentiated oligodendroglioma with almost back-to-back cells. It was difficult to distinguish from astrocytoma in infiltrat- ing areas with lower cellularity and was similar to the more "diffuse" areas of T262.

T273 A highly cellular neoplasm with more nuclear irregularity than Grade II oligodendroglioma and with nuclear pleomorphism that overlapped with that of astrocytic neoplasms. The tumor was, however, cytologically monotonous, microcystic, and densely calcified. 
T77 A classic, very well-differentiated Grade II example with back-to-back cells with perinuclear haloes.

\section{Astrocytomas}

T306 A glioma with a definite oligodendroglioma quality because of nuclear roundness and perinuclear haloes but with nuclei that were darker, slightly more pleomorphic, and more unevenly dis- tributed than in tumors classified as oligodendrogliomas (Fig. 2A). Mitoses were present.

T107 A lesion similar to T270, T281, T283, and T306, with low cellularity and perinuclear haloes but with mild variation of nuclear size and shape (Fig. 2B).

T281 A tumor with an oligodendroglioma quality because of focal round nuclei, but the latter were overall more irregular than in oligodendrogliomas.
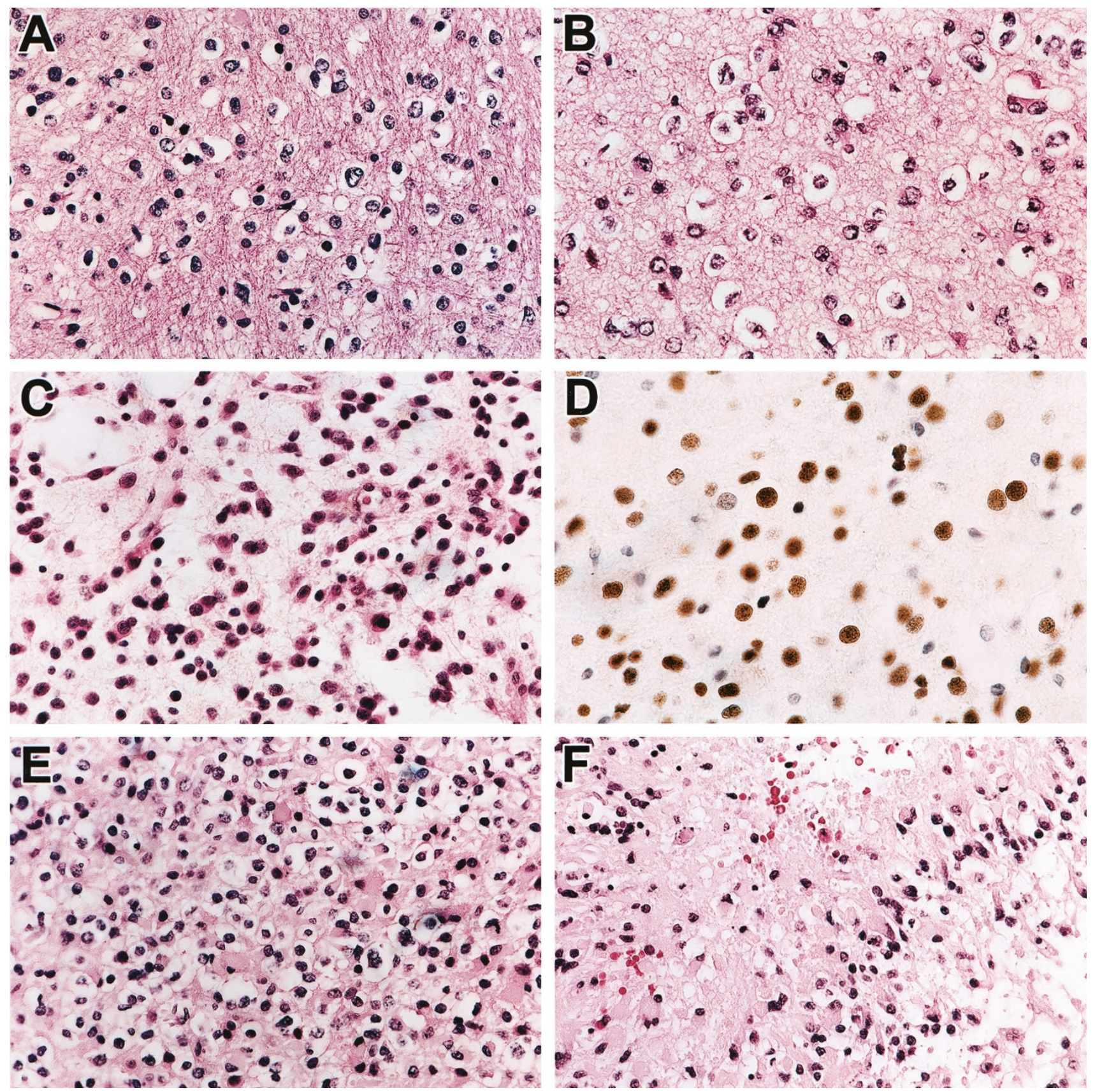

FIGURE 2. Histological and immunohistochemical features of representative cases without combined loss of chromosomes 1p and 19q. The tumors in A, Case T306, and B, Case T107, had oligodendroglioma-like qualities, largely because of the perinuclear haloes, but the nuclei were somewhat more variable in size and configuration than those in oligodendrogliomas as illustrated in Figure 1A to C. The neoplasm in (C) and (D), Case T270, had some oligodendroglioma qualities, but there was more irregularity in the shape of the nuclei than was found in the oligodendrogliomas with $1 \mathrm{p}$ and $19 \mathrm{q}$ loss. In addition, there was extensive nuclear immunoreactivity for p53 (D) as a consequence of a mutation in the gene in Exon 8 (Codon 273). The lesion in (E) and (F), Case T272, was classified histologically as a malignant mixed glioma because of cells with round nuclei and prominent perinuclear haloes $(\mathbf{E})$. Necrosis with pseudopalisading was present $(\mathbf{F})$. 
Similar to T107, T306, T270, and T383. Scattered mitoses were present.

T276 A glioma with lower grade areas with oligodendroglioma quality due largely to extreme autolytic change with prominent perinuclear haloes, but with more nuclear irregularity than oligodendrogliomas. Elsewhere, a Grade IV gemistocytic lesion with necrosis with pseudopalisading. One section studied for molecular features was more "oligodendroglioma-like" and the other more "astrocytic," i.e., the latter had true gemistocytes and necrosis with pseudopalisading.

T274 A neoplasm with variable histological appearances, i.e., in some areas a gemistocytic Grade III astrocytoma, but in others a lesion with dense masses of gemistocytes and minigemistocytes. Necrosis with focal pseudopalisading was present.

T230 A lesion with some oligodendroglioma quality but, like T306, T270, and T383, with nuclei that were darker and more irregular than in the classic well differentiated oligodendroglioma. Focal small gemistocytes and scattered mitoses were present. .

T270 A neoplasm of medium cellularity, with slightly more nuclear pleomorphism than in most oligodendrogliomas (Fig. 2C). Almost all tumor cells were immunoreactive for p53 (Fig. 2D).

T272 A "mixed" lesion with focal areas with perinuclear haloes, gemistocytes, and smaller "minigemistocytes" (Fig. 2E). Also present was a smallcell component with irregular nuclei. Focal necrosis with pseudopalisading was present (Fig. 2F).

T283 A glioma similar to T107, T283, T306, and T270, with some oligodendroglioma qualities but with nuclei that were darker and somewhat more irregular than in oligodendrogliomas. Scattered mitoses were present.

T245 A classic Grade III astrocytoma with dark angulated nuclei. Scattered mitoses were present.

T278 A classic gemistocytic astrocytoma. No mitoses were present.

T208 A Grade III astrocytoma with large gemistocytes, nuclear pleomorphism, and very focal vascular proliferation. There were focal areas with round nuclei with haloes, but the lesion lacked the cellular monotony of oligodendrogliomas.

\section{Immunohistochemistry for p53}

As is summarized in Table 1 , nuclear staining varied considerably, varying from tumors with no positive cells to those wherein all tumor cells were reactive (Fig. 2D). Reactive astrocytes and blood vessels were unstained in all cases. All six neoplasms with $1 \mathrm{p}$ and $19 \mathrm{q}$ loss were notable for a very low incidence of stained cells.
Comparative Genomic Hybridization

All six oligodendrogliomas (T277, T275, T273, T262, T220, and T77) demonstrated loss of chromosomal arms $1 p$ and 19q (Figs. 3 and 5, and Table 1). No other neoplasm, including the "malignant mixed glioma" (T272), showed this same combination loss. The two sections from T276 had identical changes.

\section{PCR-Based Microsatellite Analysis}

Six tumor specimens demonstrated homozygosity of all informative $1 p$ microsatellite markers analyzed. Based on the average high heterozygosity scores (fraction of a randomly selected population expected to be heterozygous) of the markers, this strongly suggests loss of one copy of $1 p$ (Figs. 4 and 5). Loss of $1 p$ was also detected by both FISH and CGH for these six cases. The remaining tumor specimens and the control samples demonstrated heterozygosity for at least three microsatellite markers, a finding consistent with retention of the chromosome arm.

Eleven tumor specimens demonstrated homozygosity of all informative 19q microsatellite markers analyzed. Based on the high heterozygosity score of these markers, this strongly suggests loss of one copy of 19q. For 10 of these cases, FISH and CGH also detected loss of 19q. PCR analysis for case T283 revealed considerable heterozygosity, consistent with normal 19q copy number; however, both FISH and CGH detected loss of 19q. Because FISH detected four copies of the chromosome 19 centromere, reduplication followed by loss may explain the presence of two different alleles for the $19 q$ microsatellites.

The remaining cases demonstrated heterozygosity for at least two microsatellite markers, consistent with normal 19q copy number.

The two sections from T276 had identical changes, although the heterozygosity status was indeterminate for three markers.

\section{Fluorescence In Situ Hybridization}

FISH analysis detected 6 cases with hemizygous deletion of $1 \mathrm{p} 36$ and 11 cases with hemizygous deletion of 19q13.3 (Figs. 4 and 5). All cases with deletion of 1 p36 also had concomitant deletion of 19q13.3. Based on FISH analysis, T283 exhibited tetrasomy for chromosomes 1 and 19, with relative deletion of 19q13.3 (i.e., two copies). The $1 \mathrm{p}$ and 19q copy number, as determined by FISH, was entirely consistent with that determined by CGH. The two sections from T276 had identical changes.

\section{Sequencing of $\mathrm{p} 53$}

Sequencing was successful in all cases except Case T278 and was not done for Case T107. Of the 


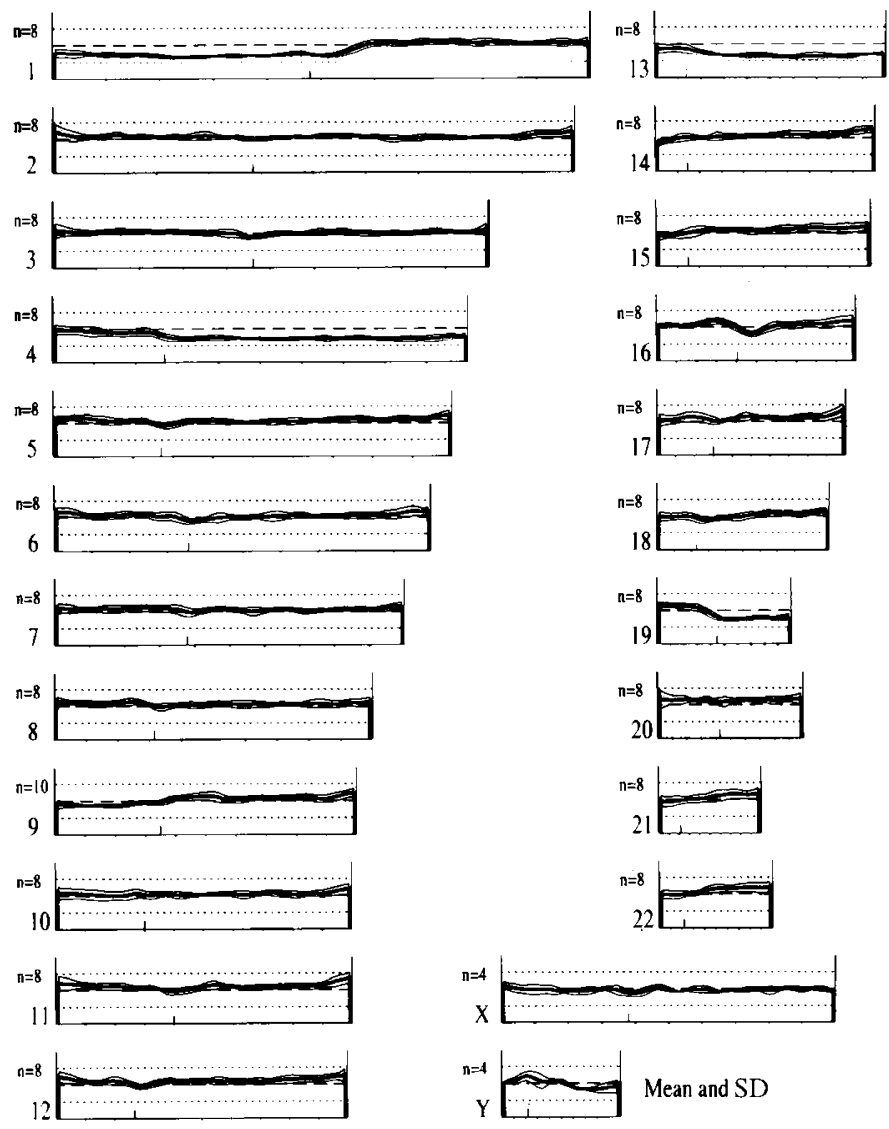

FIGURE 3. Comparative genomic hybridization profile for oligodendroglioma (Case T277). There is loss of chromosomal arms 1p and 19q. There are additional losses of $4 \mathrm{q}, 9 \mathrm{p}$, and $13 \mathrm{q}$.

16 successful cases, mutations were found in five, as is summarized in Table 1. The codons affected, and the consequences of the mutations, were as follows: T245-Exon 8 (Codon 273, C $\rightarrow$ T); T283Exon 8 (Codon 273, $\mathrm{C} \rightarrow \mathrm{T}$, homozygous); T270Exon 8 (Codon 273, homozygous); T272-Exon 8 (Codon 273, $\mathrm{C} \rightarrow \mathrm{T}$, heterozygous); and T208-Exon 7 (Codon 234, A $\rightarrow \mathrm{G}$, heterozygous) and Exon 8 (Codon 273, C $\rightarrow$ T). No p53 mutations were found in any of the six lesions with both $19 q$ and $1 p$ loss.

\section{DISCUSSION}

Although the investigation was in large part a feasibility study rather than a numerically large effort suitable for statistical analysis, all six lesions classified histopathologically as oligodendroglioma had loss of chromosomal arms $1 p$ and $19 q$ by CGH, FISH, and PCR, in accord with emerging data concerning the molecular/cytogenetic anatomy of this glioma (2-7, 9-12). These six lesions were designated oligodendroglioma using strict histological criteria, in other words, a neoplasm with a uniform population of round cells, focally at least back-to-back, with prominent perivascular haloes. Focal nuclear roundness, or halo formation, was not in itself sufficient, nor was the subjective feeling that the lesion had an oligodendro- glioma quality. Only two lesions that we believed had a histological oligodendroglial component (T208 and T272) did not have the loss of both $1 p$ and 19q, and these lesions were a somewhat ill-defined entity, in other words, "mixed glioma," in these cases malignant. The remaining lesions, of which seven had been labeled by the referring institution as oligodendroglioma or mixed glioma, lacked this pair of oligodendroglial markers. Although our study was limited, the fact that 8 of the 14 institutionally diagnosed oligodendrogliomas or mixed oligoastrocytomas lacked the $1 \mathrm{p} /$ 19q loss certainly does not dispel concerns that oligodendrogliomas are presently often overdiagnosed.

Interpretation of the results of this study must confront first the issue of whether a neoplasm is a histopathological or a molecular/cytogenetic entity, or both. The issue has arisen already in the case of mixed gliomas and malignant oligodendrogliomas, in which two dichotomous molecular/cytogenetic subtypes have been noted for what are, ostensibly, the same lesion as defined by histological criteria. Oligoastrocytomas investigated in regard to allelic loss by classic LOH methods were either those with an "oligodendroglioma" genotype, in other words, the $1 \mathrm{p} / 19 \mathrm{q}$ loss, and those with a more "astrocytic" genotype, in other words, with $\mathrm{LOH}$ of $17 \mathrm{p}$ and, often, a p53 mutation (5, $6,9)$. Two distinct molecular/cytogenetic groups have 

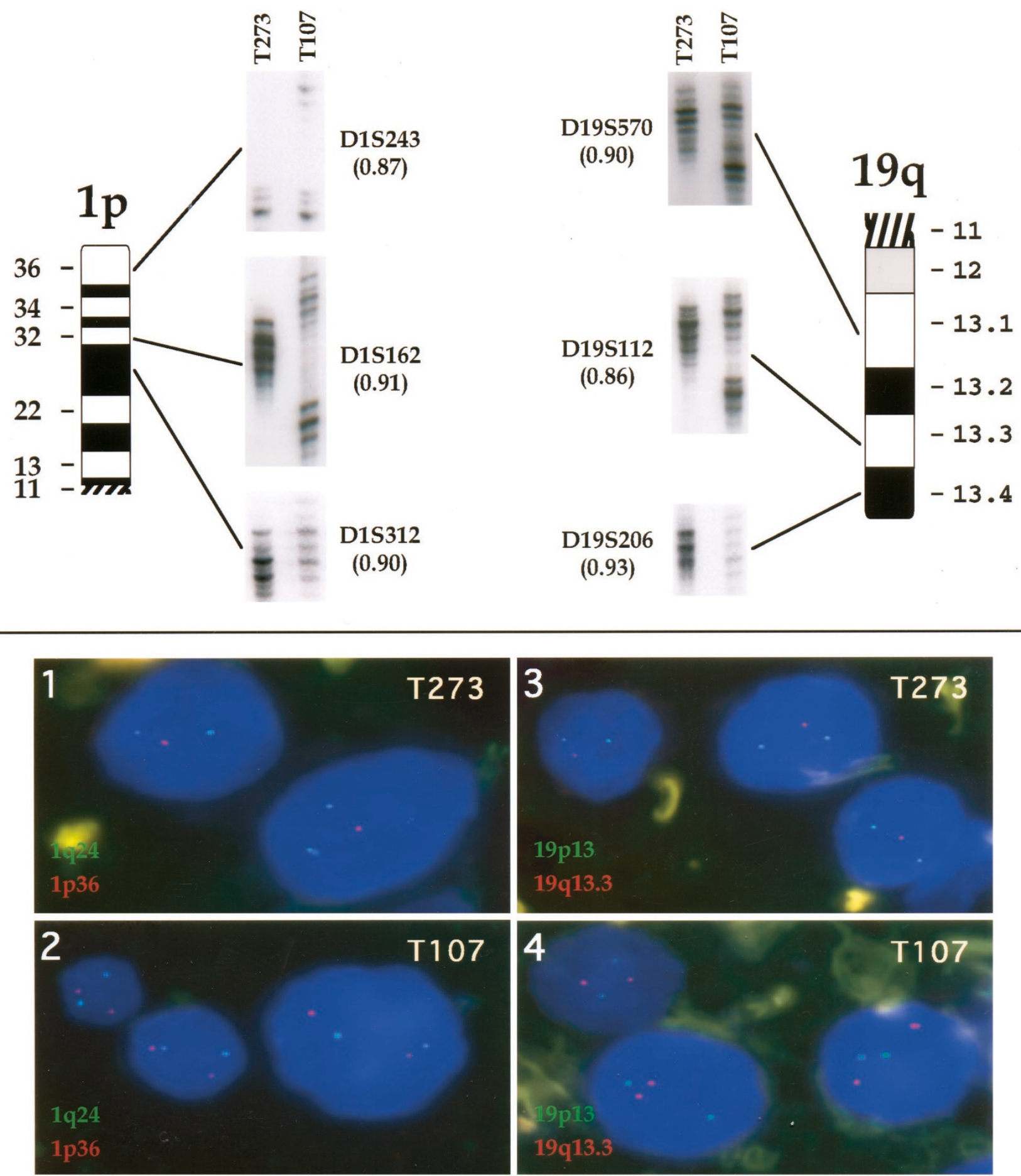

FIGURE 4. Representative microsatellite analysis (top) and representative fluorescence in situ hybridization (FISH) images (bottom) for T273 and T107. The cytogenetic location of each marker is indicated by the solid black line, and below each marker label is indicated, in parentheses, the heterozygosity score, that is, the fraction of a randomly selected population expected to be heterozygous for the marker. Each gel image depicts two lanes; the left lane represents the microsatellite allelic pattern from Tumor T273, and the right lane represents that of Case T107. For each marker illustrated, Case 273 shows amplification of a single allele (apparent homozygosity due either to chromosomal loss or homozygosity at this locus for the marker employed), and Tumor T107 shows the normal two alleles (heterozygosity). The markers employed were those unlikely to be shared by both chromosomes, that is, homozygous. The expected probability of heterozygosity in the general population, het score, ranged from .80 ( $80 \%)$ to .93 (93\%), as shown in Figure 5. FISH Image 1 contains two nuclei from Case T273 that each show two copies of the 1q24 FISH probe (blue-green) and only one copy of the 1p36 FISH probe (red), indicating hemizygous deletion of 1p36. The corresponding images for Case 107, shown in Image 2, illustrate no apparent deletion of 1p36. Similarly, for chromosome 19 analysis, Image 3 depicts hemizygous deletion of 19q13.3 for Case T273, whereas Image 4 demonstrates no apparent deletion for 19q13.3 for Case T107. Two separate regions on separate slides were studied for T276. 

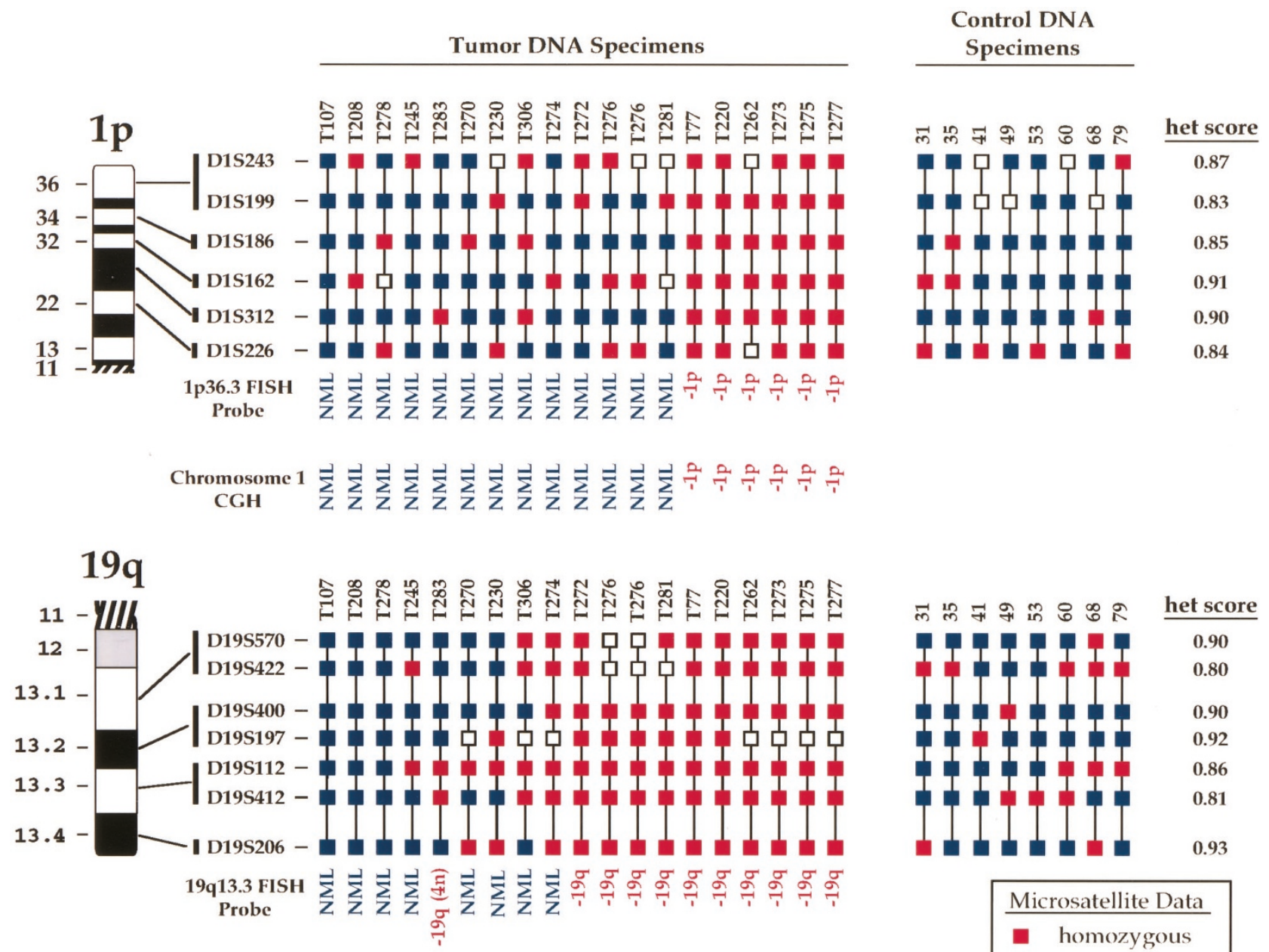

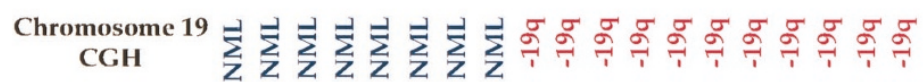

\begin{tabular}{|ll|}
\hline Microsatellite Data \\
\hline - & homozygous \\
$\square$ & heterozygous \\
$\square$ & indeterminant \\
\hline
\end{tabular}

FIGURE 5. Summary of $1 p$ and $19 q$ alterations as assessed by comparative genomic hybridization (CGH), fluorescence in situ hybridization (FISH), and polymerase chain reaction. Data points from microsatellite analyses are indicated by red squares (homozygosity), blue squares (heterozygosity), and white squares (indeterminate). NML (for the FISH and CGH results), the specimen did not demonstrate alteration of the corresponding FISH probe or chromosome arm, respectively; Het score, heterozygosity score: the fraction of a randomly selected population expected to be heterozygous for a marker; 4n (adjacent to the Tumor T283 19q13.3 FISH result), tumor was tetraploid for 19q.

been reported as well among malignant oligodendrogliomas, where microsatellite analysis was used to subclassify the neoplasms $(7,8)$. Remarkably, only those with an altered marker responded to postoperative radiotherapy and chemotherapy.

If the1p/19q combination is a marker for oligodendrogliomas, what is the nature of oligodendrogliomaappearing lesions without this signature? Our review diagnoses, cytogenetic findings, and p53 mutations suggest that most of such lesions in our study were astrocytomas. As discussed above, published studies of both mixed gliomas and malignant oligodendrogliomas have documented cases that lacked $1 p$ and $19 q$ loss. Whether these are called oligodendrogliomas or mixed gliomas depends on how an entity is defined. Clinically, however, $1 \mathrm{p}$ and 19q loss has profound implications; studies of both low- and high-grade lesions have found that oligodendrogliomas with this loss did significantly better than the "oligodendrogliomas" without the loss $(7,11)$. Loss of $1 p$ may also predict a better outcome in tumors without features of oligodendroglioma (12).

The possibility exists that abnormalities in "oligodendroglioma" genes could exist without the gross chromosomal changes detected by CGH or FISH. Thus, oligodendrogliomas may arise from point mutations or small interstitial aberrations that affect the as-yet-to-be-defined "oligodendroglioma gene or genes." Although a gene or genes on $19 q$ that is or are instrumental for the pathogenesis of oligodendroglioma have not yet been defined, the FISH probes used in the study are in a region of common deletion in 
gliomas (9), thus minimizing chances that a critical loss of $19 \mathrm{q}$ would be missed and a glioma falsely assigned to a nonoligodendroglioma category by FISH using the probes employed in these studies.

CGH, FISH, and PCR neither are simple procedures nor are commonly used in the general pathology laboratory, but they are often available in the research setting. Although CGH can be applied to paraffin-embedded tissues (33), it requires an individual skilled in chromosome identification. FISH is applied more readily but requires careful study of multiple nuclei to provide the statistically necessary number of observations. PCR approaches are optimally done together with normal DNA and tumor DNA obtained from the same patient to determine whether the germline is homozygous and, hence, whether homozygous amplicons from the tumor represent loss of heterozygosity. When control DNA is not available, as in this study, microsatellite markers can still be employed for homozygosity mapping, but the technique is more subject to contamination from normal tissue present in the tumor sample. Homozygosity mapping is a more rapid and simpler form of analysis that may be more easily applied, but the results are more difficult to interpret because reference amplicons are not available. Furthermore, PCR analysis can detect loss and reduplication, whereas FISH and CGH cannot. The frequency of these tumors with loss and reduplication is unknown, but it is very possible that the reduplicated arm also harbors the defective "glioma gene." No chromosomal loss would be apparent in this situation by either CGH or FISH.

Despite these limitations, there is no reason to assume that given advances in automation, either these or other techniques could become routine in the clinical laboratory. At a minimum, CGH, FISH, and PCR data may be used as references to standardize histological criteria. Genetic studies could well become standard in the frequently difficult distinction between oligodendroglioma and astrocytoma. The secondary component to this study was undertaken to see how immunohistochemistry for p53 and direct DNA sequencing of this gene might complement CGH and FISH in the setting of an infiltrating glioma where the cell type is at issue. Although immunohistochemical studies of p53 levels in oligodendrogliomas suggest a significant percentage of immunopositive cells $(16,17)$, it appears that mutations in Exons 5 to 8 are uncommon. Ohgaki et al. (13) recorded only 2 of 17 oligodendrogliomas with mutations. The present study is not large enough to estimate this incidence of p53 mutations, but the results are consistent with a low incidence in Grade II oligodendrogliomas. This suggests that one may be skeptical of the diagnosis of oligodendroglioma in the face of a p53 mutation or when all or most nuclei are strongly stained immunohistochemically for p53.

\section{REFERENCES}

1. Shaw EG, Scheithauer BW, O'Fallon JR, Tazelaar HD, Davis DH. Oligodendrogliomas: the Mayo Clinic experience. J Neurosurg 1992;76:428-34.

2. von Deimling A, Louis DN, von Ammon K, Petersen I, Wiestler OD, Seizinger BR. Evidence for a tumor suppressor gene on chromosome $19 q$ associated with human astrocytomas, oligodendrogliomas, and mixed gliomas. Cancer Res 1992; 52:4277-9.

3. Reifenberger J, Reifenberger G, Liu L, James CD, Wechsler W, Collins VP. Molecular genetic analysis of oligodendroglial tumors shows preferential allelic deletions on $19 \mathrm{q}$ and $1 \mathrm{p}$. Am J Pathol 1994;145:1175-90.

4. Ritland SR, Ganju V, Jenkins RB. Region-specific loss of heterozygosity on chromosome 19 is related to the morphologic type of human glioma. Genes Chromosom Cancer 1995; 12:277-82.

5. Kraus JA, Koopmann J, Kaskel P, Maintz D, Brandner S, Schramm J, et al. Shared allelic losses on chromosomes $1 \mathrm{p}$ and 19q suggest a common origin of oligodendroglioma and oligoastrocytoma. J Neuropathol Exp Neurol 1995;54:91-5.

6. Maintz D, Fiedler K, Koopmann J, Rollbrocker B, Nechev S, Lenartz D, et al. Molecular genetic evidence for subtypes of oligoastrocytomas. J Neuropathol Exp Neurol 1997;56:1098104.

7. Cairncross JG, Ueki K, Zlatescu MC, Lisle DK, Finkelstein DM, Hammond RR, et al. Specific genetic predictors of chemotherapeutic response and survival in patients with anaplastic oligodendrogliomas. J Natl Cancer Inst 1998;90:1473-9.

8. Louis DN, Cairncross JG. Specific chromosomal losses predict chemotherapeutic response and survival in patients with anaplastic oligodendrogliomas. J Neuropathol Exp Neurol 1998;57:472.

9. Smith JS, Alderete B, Minn Y, Borell TJ, Perry A, Mohapatra $\mathrm{G}$, et al. Localization of common deletion regions on $1 \mathrm{p}$ and $19 \mathrm{q}$ in human gliomas and their association with histological subtype. Oncogene 1999;18:4144-52.

10. Bigner SH, Rasheed A, Wiltshire, McLendon RE. Morphologic and molecular genetic aspects of oligodendroglial neoplasms. Neuro Oncol 1999;1:52-60.

11. Smith JS, Perry A, Borell TJ, Lee HK, O'Fallon J, Hosek SM, et al. Alterations of chromosome arms $1 \mathrm{p}$ and $19 \mathrm{q}$ as predictors of survival in oligodendrogliomas, astrocytomas, and mixed oligoastrocytomas. J Clin Oncol 2000;18:636-45.

12. Ino Y, Zlatescu MC, Sasaki H, Macdonald DR, StemmerRachamimov AO, Jhung S, et al. Long survival and therapeutic responses in patients with histologically disparate highgrade gliomas demonstrating chromosome $1 \mathrm{p}$ loss. J Neurosurg 2000;92:983-90.

13. Ohgaki H, Eibl R, Wiestler O, Yasargil M, Newcomb E, Kleihues P. P53 Mutations in nonastrocytic human brain tumors. Cancer Res 1991;51:6202-5.

14. Tong CY, Ng H-K, Pang JC, Hui AB, Ko HC, Lee JC. Molecular genetics of non-astrocytic gliomas. Histopathology 1999;34: $331-41$.

15. von Deimling A, Fimmers R, Schmidt MC, Bender B, Fassbender F, Nagel J, et al. Comprehensive allelotype and genetic analysis of 466 human nervous system tumors. J Neuropathol Exp Neurol 2000;59:544-58.

16. Kros JM, Godschalk JJCJ, Krishnadath KK, Van Eden CG. Expression of p53 in oligodendroglioma. J Pathol 1993;171: 285-90.

17. Pavelic J, Hlavka V, Poljak M, Gale N, Pavelic K. p53 immunoreactivity in oligodendrogliomas. J Neurooncol 1994;22: $1-6$. 
18. Kleihues P, Cavenee WK. World health organization classification of tumours. Pathology and genetics. Tumours of the central nervous system. Lyon, France: IARC Press; 2000.

19. Patel A, van Meyel DJ, Mohapatra G, Bollen A, Wrensch M, Cairncross JG, et al. Gliomas in families: chromosomal analysis by comparative genomic hybridization. Cancer Genet Cytogenet 1998;100:77-83.

20. Telenius H, Carter NP, Bebb CE, Nordenskjold M, Ponder BA, Tunnacliffe A. Degenerate oligonucleotide-primed PCR: general amplification of target DNA by a single degenerate primer. Genomics 1992;13:718-25.

21. Mohapatra G, Kim DH, Feuerstein BG. Detection of multiple gains and losses of genetic material in ten glioma cell lines by comparative genomic hybridization. Genes Chromosom Cancer 1995;13:86-93.

22. Piper J, Rutovitz D, Sudar D, Kallioniemi A, Kallioniemi OP, Waldman FM, et al. Computer image analysis of comparative genomic hybridization. Cytometry 1995;19:10-26.

23. Banerjee SK, Makdisi WF, Weston AP, Mitchell SM, Campbell DR. Microwave-based DNA. Extraction from paraffinembedded tissue for PCR amplification. Biotechniques 1995; 18:768-70, 772-3.

24. Ashworth LK, Batzer MA, Brandriff B, Branscomb E, de Jong $\mathrm{P}$, Garcia E, et al. An integrated metric physical map of human chromosome 19. Nat Genet 1995;11:422-7.

25. Collins A, Keats BJ, Dracopoli N, Shields DC, Morton NE. Integration of gene maps: chromosome 1. Proc Natl Acad Sci USA 1992;89:4598-602.

26. GDB(TM) Human Genome Database (database online). Bal- timore, MD: Johns Hopkins University; 1990. Available at www.gdb.org/.

27. Murray JC, Buetow KH, Weber JL, Ludwigsen S, Scherpbier-Heddema T, Manion F, et al. A comprehensive human linkage map with centimorgan density. Cooperative Human Linkage Center (CHLC). Science 1994;265: 2049-54.

28. Rosenberg JE, Lisle DK, Burwick JA, Ueki K, von Deimling A, Mohrenweiser HW, et al. Refined deletion mapping of the chromosome $19 \mathrm{q}$ glioma tumor suppressor gene to the D19S412-STD interval. Oncogene 1996;13:2483-5.

29. Smith JS, Jenkins RB. Loss of heterozygosity mapping of the chromosome 19q- arm in human gliomas [abstract]. FASEB J 1997;11:A45131.

30. Jenkins RB, Qian J, Lieber MM, Bostwick DG. Detection of c-myc oncogene amplification and chromosomal anomalies in metastatic prostatic carcinoma by fluorescence in situ hybridization (FISH). Cancer Res 1997;57: 524-31.

31. Jenkins RB, Qian J, Lee HK, Huang H, Hirasawa K, Bostwick DG, et al. A molecular cytogenetic analysis of $7 \mathrm{q} 31$ in prostate cancer. Cancer Res 1998;58:759-66.

32. Brat DJ, James CD, Jedlicka AE, Connolly DC, Chang E, Castellani RJ, et al. Molecular genetic alterations in radiation- induced astrocytomas. Am J Pathol 1999;154:1431-38.

33. Isola J, De Vries S, Chu L, Ghazvini S, Waldman F. Analysis of changes in DNA sequence copy number by comparative genomic hybridization in archival paraffin-embedded tumor samples. Am J Pathol 1994;145:1301-8. 